\title{
Susceptibilidad antimicrobiana de Streptococcus pneumoniae colonizante de nasofaringe en niños colombianos con neumonía1
}

\author{
Aura Lucía Leal ${ }^{2}$ y Elizabeth Castañeda²
}

\begin{abstract}
RESUMEN Streptococcus pneumoniae es uno de los principales agentes causales de infección respiratoria aguda (IRA) en niños y su resistencia a antibióticos se ha incrementado en todo el mundo. En este estudio se determinaron los patrones de susceptibilidad a antimicrobianos de S. pneumoniae colonizante de las vías respiratorias altas en 272 niños hospitalizados por neumonía en dos hospitales de Santafé de Bogotá. Se aisló S. pneumoniae en 114 pacientes (42\%). Se observó susceptibilidad disminuida a la penicilina en 19 aislamientos (17\%), con sensibilidad intermedia en 12 (11\%) y franca resistencia en 7 (6\%). Solo 1 de los 19 aislamientos resistentes a penicilina mostró también resistencia a la ceftriaxona. Se observó sensibilidad disminuida a la eritromicina en 3 aislamientos (3\%), al cloranfenicol en $6(5 \%)$ y al cotrimoxazol (trimetoprima + sulfametoxazol) en 46 (40\%). Se encontró multirresistencia en 7 aislamientos (6\%). El serotipo con sensibilidad disminuida a la penicilina que se halló con mayor frecuencia fue el $23 F(68,4 \%)$. Se observó una asociación entre la edad, el uso previo de antibióticos y la colonización con S. pneumoniae con susceptibilidad disminuida a la penicilina o multirresistencia. Este estudio confirma la presencia de resistencia antimicrobiana de S. pneumoniae en Colombia y resalta la importancia del uso racional de los antibióticos y de la implementación de la vigilancia epidemiológica sobre este agente.
\end{abstract}

En los países en desarrollo la infección respiratoria aguda (IRA), principalmente la neumonía, es responsable de aproximadamente 4,5 millones de muertes cada año, especialmente en niños menores de 5 años (1). En la mayoría de los episodios de IRA el agente causal es un virus, pero estas

\footnotetext{
1 Próximamente se publicará en esta revista una versión en inglés de este artículo.

2 Grupo de Microbiología, Instituto Nacional de Salud, Santafé de Bogotá, Colombia. La correspondencia debe enviarse a: Elizabeth Castañeda, Laboratorio de Microbiología, Instituto Nacional de Salud, Avenida Eldorado, Carrera 50, Santafé de Bogotá, Colombia (Tel. [57-1] 222-0577, ext. 445 ó 446; fax [57-1] 222-0194).
}

infecciones son generalmente autolimitadas. La neumonía bacteriana, aunque menos frecuente, se asocia con mayor riesgo de complicaciones y alta mortalidad (2).

Los dos agentes bacterianos aislados con mayor frecuencia en niños con neumonía son Streptococcus pneumoniae (neumococo) y Haemophilus influenzae (3). Teniendo en cuenta que estos microorganismos pueden colonizar las vías respiratorias altas, es posible que la neumonía sea producida por la aspiración de secreciones, especialmente si existen factores predisponentes a la infección bacteriana como desnutrición, infecciones virales $\mathrm{u}$ otros facto- res que alteren la respuesta inmunitaria (4). La colonización de las vías aéreas altas por $S$. pneumoniae puede producirse en los primeros días de vida y esta colonización temprana se relaciona con la convivencia en familias numerosas y aumenta el riesgo de padecer la enfermedad (5).

El diagnóstico etiológico de la neumonía neumocócica no es fácil ya que el hemocultivo de $S$. pneumoniae posee una baja sensibilidad y otros procedimientos invasores, como la punción pulmonar, implican un riesgo significativo para el paciente. Por tanto, el protocolo desarrollado por la Organización Mundial de la Salud (OMS) 
para el manejo de la IRA contempla el uso empírico de agentes antimicrobianos como la penicilina procaínica, la ampicilina, la amoxicilina y el cotrimoxazol (trimetoprima + sulfametoxazol) $(6,7)$.

Las notificaciones de resistencia de S. pneumoniae a diversos antimicrobianos son cada vez más frecuentes en todo el mundo. En España y Hungría se ha informado de resistencia a la penicilina en el 44 y 57\%, respectivamente, de los aislamientos; la resistencia a cotrimoxazol y cloranfenicol también ha sido frecuente $(8,9)$. La edad, los serotipos, el tiempo de colonización, la rápida recolonización después de un tratamiento, el uso previo de antibióticos y la hospitalización previa han sido los factores que se han hallado asociados con la presencia de gérmenes aislados resistentes (8).

Estudios realizados en Pakistán indican que, en los niños enfermos, los serotipos de S. pneumoniae aislados de las vías respiratorias coinciden con los recuperados en el hemocultivo. Coinciden asimismo, hasta en $98 \%$ de los casos, los patrones de susceptibilidad a antimicrobianos de estos aislamientos (10). Por tanto, en niños con neumonía, los aislamientos de S. pneumoniae de nasofaringe podrían reflejar el patrón de resistencia antimicrobiana de los gérmenes que causan enfermedad invasora y serían útiles para realizar programas de vigilancia epidemiológica en un área determinada. Esto a su vez permitiría hacer un manejo terapéutico más racional (11).

El objetivo de este trabajo fue determinar: a) la frecuencia de colonización nasofaríngea por S. pneumoniae en niños con neumonía; b) los patrones de resistencia antimicrobiana de los aislamientos correspondientes frente a los antibióticos recomendados en el programa de IRA; c) los serotipos de los gérmenes aislados resistentes a la penicilina hallados; y d) algunas características asociadas con el patrón de resistencia antimicrobiana. La evaluación de los datos obtenidos permitiría determinar la utilidad de los aislamientos nasofaríngeos para realizar un programa de vigilancia.

\section{MATERIAL Y MÉTODOS}

Pacientes. Se estudiaron 272 pacientes, menores de 5 años, que fueron ingresados al Hospital Infantil Lorencita Villegas de Santos o al hospital La Misericordia de la ciudad de Santafé de Bogotá con diagnósticos de neumonía o neumonía grave, según los criterios de la OMS. El criterio de la OMS para el diagnóstico de neumonía es la frecuencia respiratoria alta (más de 60 por minuto en menores de 2 meses, más de 50 en niños de 2 meses a 1 año, y más de 40 en mayores de 1 año); el diagnóstico de neumonía grave exige además la presencia de tiraje (6). Como criterio de inclusión adicional se consideró una evolución clínica de menos de 15 días y se excluyeron los pacientes con cuadros de estridor o crup (laringotraqueobronquitis aguda).

En cada caso, se consignaron en un formulario los datos de identificación, edad, uso previo de antibióticos, frecuencia respiratoria, presencia de tiraje y diagnóstico clínico. El estudio se llevó a cabo de noviembre de 1993 a octubre de 1994.

Muestra, aislamiento e identificación. A cada paciente se le tomó una muestra de secreción nasofaríngea, con escobillón flexible de dacrón, la cual se sembró directamente en un medio de agar tripticasa soya, adicionado con sangre de cordero al 5\% y gentamicina en una concentración de $5 \mu \mathrm{g} / \mathrm{mL}$ (12). Las muestras se incubaron a $37^{\circ} \mathrm{C}$ durante $20-24$ horas en una atmósfera con $2-5 \%$ de $\mathrm{CO}_{2}$ (método de jarra con vela).

Para la identificación de S. pneumoniae se tuvieron en cuenta las características macroscópicas y de alfahemólisis de la colonia, la morfología microscópica con tinción de Gram, la susceptibilidad a la optoquina y la solubilidad en bilis (13).

Susceptibilidad antimicrobiana. La susceptibilidad a la penicilina se determinó mediante la técnica de difusión en disco (Kirby-Bauer), con el empleo de agar Müeller-Hinton con sangre de cordero al 5\% y discos de oxacilina de $1 \mu \mathrm{g}$ (prueba tamiz). Esta misma técnica se utilizó para determinar la susceptibilidad a la eritromicina, con discos de $15 \mu \mathrm{g}$, y al cloranfenicol, con discos de $30 \mu \mathrm{g}$. Todos los procedimientos se realizaron e interpretaron teniendo en cuenta los parámetros establecidos por el comité estadounidense de estándares de laboratorio (NCCLS) (14). Como cepa control se utilizó S. pneumoniae ATCC 49619.

En los aislamientos que presentaron susceptibilidad disminuida a la penicilina (halo de inhibición con la oxacilina de menos de $20 \mathrm{~mm}$ de diámetro) se determinó la concentración inhibitoria mínima (CIM) para penicilina y ceftriaxona. También se determinó mediante CIM la susceptibilidad de todos los aislamientos frente a cotrimoxazol (15). En todos los casos se utilizó el método de microdilución con caldo Müeller-Hinton y sangre lisada de caballo al $5 \%$ y se siguieron los protocolos establecidos por el NCCLS (16).

Serotipificación. Los aislamientos que presentaron susceptibilidad disminuida a la penicilina y multirresistencia (definida como resistencia a tres o más antimicrobianos) ${ }^{3}$ fueron serotipificados por medio de la reacción quellung utilizando el panel de doce antisueros del Statens Seruminstitut de Copenhague (17).

Análisis de los datos. Se calculó la frecuencia (prevalencia) de resistencia de S. pneumoniae resistente en cada una de las categorías de interés y la razón de prevalencias (RP) correspondiente. Los intervalos de confianza (IC) de $95 \%$ para la RP se determinaron mediante la versión 6 del programa Epi Info (18).

\footnotetext{
3 A estos efectos, la combinación trimetroprima + sulfametoxazol (cotrimoxazol) se consideró como un solo antimicrobiano.
} 


\section{RESULTADOS}

Se aisló S. pneumoniae en 114 de los 272 pacientes estudiados, lo cual representa un porcentaje mínimo de colonización de $42 \%$. De los pacientes colonizados, $76(67 \%)$ fueron del género masculino y 38 (33\%) del femenino; 94 (82\%) fueron menores de 3 años, y de estos, 57 (61\%), menores de un año. La mediana de edad de los portadores de $S$. pneumoniae fue 1,4 años y la mayor frecuencia de colonización se encontró a los 6 meses.

Los aislamientos de S. pneumoniae se obtuvieron a lo largo de todo el año de estudio. Sin embargo, el mayor número de aislamientos (65\%) fue recolectado durante el período de mayo a septiembre de 1994 (figura 1).

En 36 casos de los 114 (31\%) pudo determinarse que habían recibido terapia antibiótica. El antibiótico previamente utilizado había sido un betalactámico en 32 pacientes (89\%) (amoxicilina en 25 casos, penicilina $G$ en 4 , amoxicilina y penicilina $G$ en 1 y cefalosporina de segunda generación en 2). Otros 75 pacientes no habían recibido antibióticos previamente y en 3 casos no se pudo determinar si los habían recibido o no. El diagnóstico de neumonía grave se estableció en 79 pacientes $(69 \%)$.

Mediante la prueba tamiz con oxacilina se detectaron 19 aislamientos (17\%) de S. pneumoniae con sensibilidad disminuida a la penicilina. Cuando se determinó la CIM se hallaron 12 aislamientos (11\%) con resistencia intermedia y $7(6 \%)$ con resistencia franca. Solo una de las cepas resistentes presentó también resistencia a la ceftriaxona. Se encontró susceptibilidad intermedia al cotrimoxazol en 21 aislamientos (18\%) y resistencia en 25 (22\%). Se halló susceptibilidad intermedia a la eritromicina en 2 aislamientos $(2 \%)$ y resistencia franca en uno (1\%). Hubo resistencia al cloranfenicol en 6 aislamientos (5\%) (cuadro 1).

De los 19 aislamientos con baja sensibilidad a la penicilina, 13 correspondieron al serotipo 23F, otros 2 al 14, 1 al 19F y 3 no fueron serotipificables. El serotipo más frecuente en los aislamientos multirresistentes (baja sensi-
FIGURA 1. Distribución temporal de los aislamientos de S. pneumoniae colonizador de nasofaringe en 114 pacientes pediátricos hospitalizados por neumonía en Santafé de Bogotá

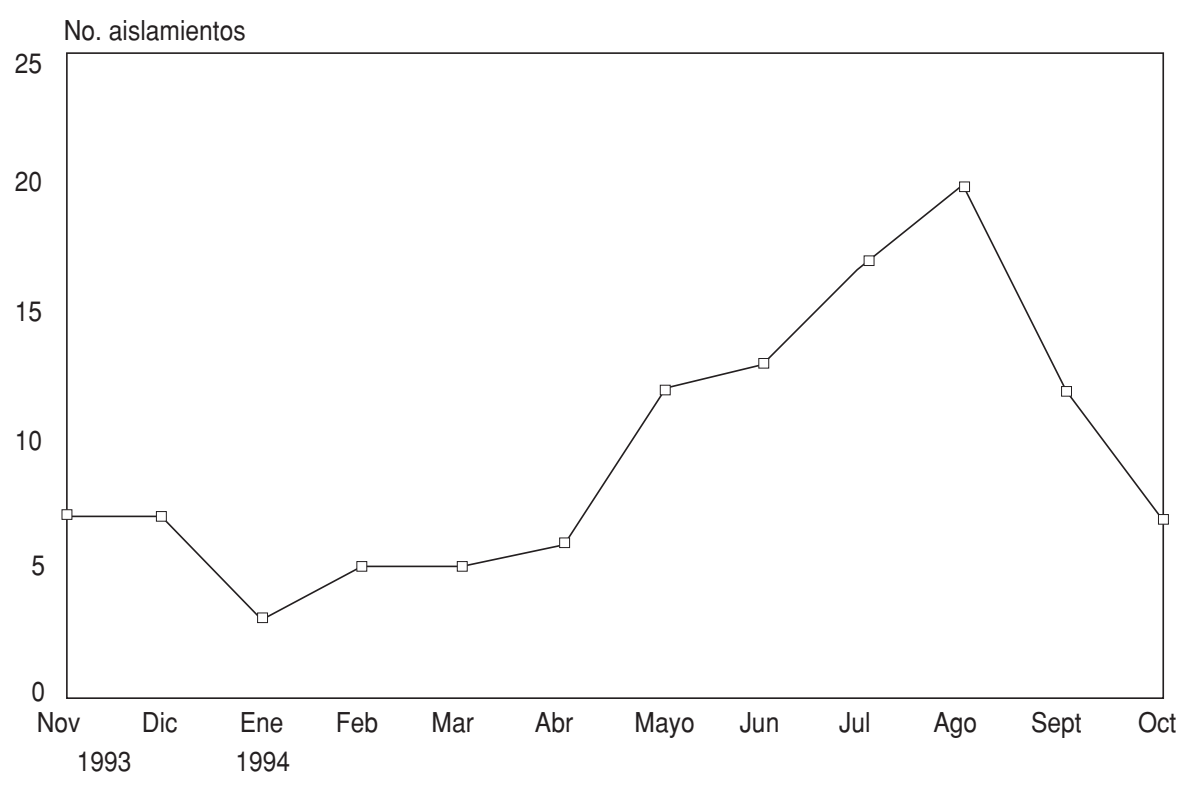

bilidad frente a tres o más antimicrobianos) fue el 23F (6 de 7 aislamientos) (cuadro 2).

Se halló sensibilidad disminuida por lo menos a un antimicrobiano en 48 aislamientos $(42 \%)$. Todos los aislamientos resistentes a la penicilina lo fueron también a cotrimoxazol. Hubo multirresistencia en 7 aislamientos $(6 \%)$; en 5 de estos hubo franca resistencia frente a todos los antimicrobianos utilizados en la prueba de sensibilidad. Todos los aislamientos multirresistentes presentaron resistencia tanto a penicilina como a cotrimoxazol. El patrón de multirresistencia
CUADRO 1. Susceptibilidad antimicrobiana de aislamientos nasofaríngeos de Streptococcus pneumoniae en 114 pacientes pediátricos hospitalizados por neumonía en Santafé de Bogotá, 1994

\begin{tabular}{|c|c|c|c|c|c|c|}
\hline \multirow{2}{*}{$\begin{array}{c}\text { Agente } \\
\text { antimicrobiano }\end{array}$} & \multicolumn{2}{|c|}{$\begin{array}{l}\text { Aislamientos } \\
\text { sensibles }\end{array}$} & \multicolumn{2}{|c|}{$\begin{array}{l}\text { Sensibilidad } \\
\text { intermedia }\end{array}$} & \multicolumn{2}{|c|}{$\begin{array}{c}\text { Aislamientos } \\
\text { resistentes }\end{array}$} \\
\hline & No. & $\%$ & No. & $\%$ & No. & $\%$ \\
\hline Penicilina ${ }^{a}$ & 95 & 83 & 12 & 11 & 7 & 6 \\
\hline Ceftriaxona ${ }^{b}$ & 18 & 95 & 0 & 0 & 1 & 5 \\
\hline Cotrimoxazol $^{c}$ & 68 & 60 & 21 & 18 & 25 & 22 \\
\hline Eritromicina $^{d}$ & 111 & 97 & 2 & 2 & 1 & 1 \\
\hline Cloranfenicol $^{\mathrm{e}}$ & 108 & 95 & 0 & 0 & 6 & 5 \\
\hline
\end{tabular}

a Concentración inhibitoria mínima $(\mathrm{CIM})<0,06 \mu \mathrm{g} / \mathrm{mL}$ en los aislamientos sensibles, entre 0,1 y $1 \mu \mathrm{g} / \mathrm{mL}$ en los de susceptibilidad intermedia y $>2 \mu \mathrm{g} / \mathrm{mL}$ en los resistentes.

${ }^{\mathrm{b}} \mathrm{CIM}<0,25 \mu \mathrm{g} / \mathrm{mL}$ en los aislamientos sensibles, de 0,5 a $1 \mu \mathrm{g} / \mathrm{mL}$ en los de sensibilidad intermedia y $>2 \mu \mathrm{g} / \mathrm{mL}$ en los resistentes.

${ }^{c} \mathrm{CIM}$ (de trimetoprima y sulfametoxazol) $<0,5 / 9,5 \mu \mathrm{g} / \mathrm{mL}$ en los aislamientos sensibles, de $1 / 19$ a $2 / 38 \mu \mathrm{g} / \mathrm{mL}$ en los de susceptibilidad intermedia $y>4 / 76 \mu \mathrm{g} / \mathrm{mL}$ en los resistentes.

d Interpretación de la difusión en disco (Kirby-Bauer): más de $21 \mathrm{~mm}$ en los aislamientos sensibles, de 16 a $20 \mathrm{~mm}$ en los de sensibilidad intermedia y menos de $15 \mathrm{~mm}$ en los resistentes.

e Interpretación de la difusión en disco (Kirby-Bauer): más de $21 \mathrm{~mm}$ en los aislamientos sensibles, de 18 a $20 \mathrm{~mm}$ en los de sensibilidad intermedia y menos de $17 \mathrm{~mm}$ en los resistentes. 
CUADRO 2. Serotipos de Streptococcus pneumoniae asociados con el patrón de resistencia

\begin{tabular}{lcl}
\hline \multicolumn{1}{c}{ Serotipo } & $\begin{array}{c}\text { No. cepas } \\
\text { aisladas }\end{array}$ & \multicolumn{1}{c}{ Patrón de resistencia } \\
\hline $23 \mathrm{~F}$ & 1 & $\begin{array}{c}\text { penicilina, ceftriaxona, cloranfenicol, cotrimoxazol } \\
\text { (trimetoprima + sulfametoxazol) } \\
\text { penicilina, eritromicina, cotrimoxazol } \\
\end{array}$ \\
& 1 & penicilina, cloranfenicol, cotrimoxazol \\
& 4 & penicilina, cotrimoxazol \\
14 & 7 & penicilina, cotrimoxazol \\
$19 \mathrm{~F}$ & 2 & penicilina, eritromicina, cotrimoxazol \\
No serotipificable & 1 & \\
Total & 3 & \\
\hline
\end{tabular}

más frecuente - a penicilina, cloranfenicol y cotrimoxazol- se presentó en 4 de los 7 aislamientos (cuadro 2).

En el grupo de 19 pacientes portadores de S. pneumoniae con sensibilidad disminuida a la penicilina o multirresistencia, 12 fueron niños y 7 niñas; 5 eran menores de 1 año, 8 tenían entre 1 y 2 años y 6 de 3 a 5 años.

De los 57 pacientes menores de 1 año, 5 estaban colonizados por S. pneumoniae resistente a la penicilina o multirresistente. De igual manera lo estaban 8 de los 37 niños de 1 ó 2 años y 6 de los 20 niños entre 3 y 5 años. Se encontró una asociación entre la edad y la presencia de cepas con baja sensibilidad a la penicilina o multirresistencia. Comparado con los menores de 1 año, el grupo de 1 a 2 años tenía una prevalencia 2,4 veces mayor de aislamientos resistentes (IC95\%: 0,9 a 7,0) y en el grupo de 3 a 5 años la prevalencia fue 3,4 veces mayor (IC95\%: 1,2 a $10,0)$. La tendencia al aumento del porcentaje de aislamientos resistentes con la edad fue estadísticamente significativa $(P=0,02)$ (cuadro 3 ).

De los 36 pacientes que habían recibido antibióticos previamente, 9 se encontraban colonizados con S. pneumoniae con resistencia a la penicilina. De los 75 que no recibieron antibióticos solo 9 fueron portadores de aislamientos resistentes. Por consiguiente, el riesgo de portar S. pneumoniae resistente fue 2,1 veces mayor (IC95\%: $0,9$ a 4,8$)$ en los niños a los que se habían administrado antibióticos previamente (cuadro 3).
En el grupo colonizado por S. pneumoniae con resistencia antimicrobiana a la penicilina o multirresistencia, el diagnóstico clínico más común fue la neumonía grave (17 de 19 casos). La frecuencia de aislamientos con resistencia fue 3,8 veces mayor en los casos de neumonía grave (IC95\%: 0,9 a 15,4) (cuadro 3).

\section{DISCUSIÓN}

La colonización de las vías respiratorias altas por $S$. pneumoniae se observa con frecuencia, especialmente en niños, pero los porcentajes de colobaja sensibilidad a la penicilina

${ }^{\mathrm{b}} \chi^{2}$ tendencia lineal, $P<0,05$.

c $P=0,02$. nización varían mucho según diferentes estudios. La relación de la colonización invasora con los aislamientos se ha confirmado en algunos casos (10, 11). Dadas las dificultades para establecer el diagnóstico etiológico en las neumonías, los aislamientos nasofaríngeos serían de utilidad para conocer los patrones de susceptibilidad antimicrobiana en un área definida y podrían predecir el comportamiento de los aislamientos responsables de cuadros invasivos en la misma zona (19).

En este estudio el porcentaje de colonización que se encontró en niños hospitalizados por neumonía fue similar a los notificados por Mogdasy et al. en el Uruguay (20) y Hendley et al. en una comunidad del estado norteamericano de Virginia (21). Sin embargo, estudios realizados en otros países muestran mayores porcentajes de colonización en niños con neumonía, por ejemplo $51 \%$ en Filipinas y $98 \%$ en Pakistán (11, 22). En Papua Nueva Guinea se han documentado porcentajes de colonización del $100 \%$ a los 3 meses de edad (23). Aunque hasta ahora no se han publicado estudios de colonización realizados en niños colombianos con neumonía, en un trabajo reciente se halló S. pneumoniae en $46 \%$ de los niños de una guardería de Santafé de Bogotá (24). Los datos de este estudio confir-

CUADRO 3. Características de los pacientes, prevalencia y razón de prevalencias (con su intervalo de confianza de 95\%) de colonización por cepas de Streptococcus pneumoniae de

\begin{tabular}{cccc}
\hline $\begin{array}{c}\text { Grupo de } \\
\text { pacientes }\end{array}$ & $\begin{array}{c}\text { Prevalencia de } \\
\text { cepas resistentes }\end{array}$ & $\begin{array}{c}\text { Razón de } \\
\text { prevalencias }\end{array}$ & IC95\% \\
\hline Sexo ô & $12 / 76$ & 0,9 & 0,37 a 2,00 \\
क & $7 / 38$ & 1,0 & \\
Edad < 1 año & $5 / 57$ & $1,0^{\mathrm{b}}$ & 0,9 a 7,0 \\
1 a 2 años & $8 / 37$ & 2,5 & 1,2 a $10,0^{\mathrm{c}}$ \\
3 a 5 años & $6 / 20$ & 3,5 & 0,9 a 4,8 \\
Administración previa de antibióticos & & & \\
Sí & $9 / 36$ & 2,1 & \\
No & $9 / 75$ & 1,0 & \\
No determinada & $1 / 3$ & & 0,9 a 15,4 \\
Diagnóstico clínico & & & \\
Neumonía & $2 / 35$ & 1,0 & \\
Neumonía grave & $217 / 79$ & 3,8 & \\
\hline
\end{tabular}

a La categoría de referencia es aquella en la que la razón de prevalencias es 1,0. 
man que la mayor proporción de portadores de neumococo se halla en menores de 2 años (23).

Los aislamientos de $S$. pneumoniae resistentes a la penicilina y a otros antimicrobianos son cada vez más frecuentes. En España, Hungría y Sudáfrica se han hallado porcentajes muy elevados de resistencia a la penicilina tanto en aislamientos colonizantes como invasivos $(9,25)$. El porcentaje de sensibilidad disminuida a la penicilina encontrado en este estudio (17\%) es importante y es mayor que los notificados en Uruguay (3\%) (20), Pakistán (12\%) (11) y Sudáfrica (12\%) (26).

Los serotipos 6, 19, 23 y 14 se han relacionado con resistencia a la penicilina o multirresistencia. Estos serotipos representan $60 \%$ de los aislamientos durante los primeros 2 años de vida $(5,21)$. Un clon del serotipo $23 \mathrm{~F}$ con resistencia a la penicilina y a otros antimicrobianos que se halló primero en España, se encontró luego en Sudáfrica y en los Estados Unidos $(27,28)$. En este estudio $68 \%$ de los aislamientos resistentes a la penicilina fueron serotipo 23F. Esto coincide con lo hallado en México, donde este serotipo se ha encontrado con mayor frecuencia en aislamientos multirresistentes (27\%) (29).

En diversos estudios se han hallado diferencias importantes entre los porcentajes de colonización y los serotipos de los aislamientos de $S$. pneumoniae en niños sanos y en niños con neumonía. En Uruguay, Mogdasy et al. encontraron $15 \%$ de colonización en niños sanos y $42 \%$ en niños con neumonía (20). Asimismo se informan mayores porcentajes de resistencia en los aislamientos de portadores sanos que en los aislamientos invasores (29). Si se compara el porcentaje de resistencia a la penicilina encontrado en este estudio $(17 \%)$ con el observado en aislamientos invasivos de neumococo en el Protocolo Nacional de Serotipificación de S. pneumoniae que se realizó en Colombia (12\%) el primero parece más alto (datos inéditos). Sin embargo, esta diferencia puede ser aparente, ya que la sensibilidad del hemocultivo, que es la técnica utilizada en el Protocolo Nacional de Serotipificación, es mucho menor que la del cultivo de la secreción nasofaríngea $(1,3)$. Es probable que la proporción de resistencia en los aislamientos nasofaríngeos refleje más exactamente el comportamiento de S. pneumoniae circulante.

Llama la atención en los resultados de este estudio el elevado porcentaje $(40 \%)$ de S. pneumoniae colonizantes con susceptibilidad disminuida al cotrimoxazol, con una proporción notable de resistencia franca $(22 \%)$ y frecuente presencia simultánea de resistencia al cotrimoxazol y la penicilina. En los últimos 15 años se han hallado porcentajes de resistencia de S. pneumoniae al cotrimoxazol del 18 a $40 \%$ en diversos países $(20,25,30,31)$. Por tanto, sería importante reconsiderar el uso de este agente, de primera elección en el manejo de la neumonía, según los protocolos del programa de IRA de la OMS.

La resistencia a eritromicina y a cloranfenicol se encontró en pocos casos, lo que concuerda con datos de Estados Unidos y Australia $(32,33)$. Por tanto, parece ser que la resistencia a estos dos agentes no es todavía un problema en nuestro medio si se compara con países como Francia, donde la resistencia a los macrólidos llega a 29\% (34) o, como España, donde se ha hallado 30\% de resistencia al cloranfenicol (35). Por tanto, en Colombia el empleo de eritromicina y cloranfenicol en neumonías infantiles parece recomendable.

Entre los factores que se han relacionado con la adquisición o infección con cepas resistentes está la exposición previa a agentes antimicrobianos (26, 36). En Sudáfrica el uso previo de penicilina y cloranfenicol se ha hallado asociado con proporciones significativamente más altas de portadores de cepas resistentes a esos dos antimicrobianos (30). En esta investigación, el uso previo de antibióticos mostró una asociación significativa con la presencia de resistencia en $S$. pneumoniae y los antibióticos utilizados con mayor frecuencia fueron los betalactámicos. Estos datos concuerdan con los informados en la literatura $(26,30)$.

Los datos obtenidos en este trabajo concuerdan con los de otros trabajos publicados e indican una asociación importante entre la edad, el uso previo de antibióticos y la colonización por S. pneumoniae con susceptibilidad antimicrobiana disminuida. Igualmente existe una asociación entre los aislamientos resistentes y el diagnóstico de neumonía grave. El tamaño de nuestra muestra no fue muy grande y la asociación no fue estadísticamente significativa en todos los casos.

Estudios similares en otras regiones del país permitirían conocer mejor la magnitud del problema de la resistencia de S. pneumoniae a antimicrobianos, establecer un verdadero programa de vigilancia y utilizar más racionalmente los agentes antimicrobianos de uso habitual frente a este importante patógeno.

Agradecimiento. Las autoras expresan su agradecimiento a Orlando Castillo y a María Claudia Vela, del Instituto Nacional de Salud (INS) de Colombia, por su colaboración en el desarrollo del estudio; a Fernando de la Hoz, también del INS, por su contribución al análisis de los datos; a Daniel Kertesz, de los Laboratorios del Centro de Control de Enfermedades de Canadá, por la revisión del manuscrito; y al personal de los hospitales Lorencita Villegas de Santos y La Misericordia por su apoyo en el desarrollo de la investigación. 
1. Berman S. Epidemiology of acute respiratory infections in children of developing countries. Rev Infect Dis 1991;13(6):454-462.

2. Shann F. Etiology of severe pneumonia in children in developing countries. Pediatr Infect Dis J 1986;5(2):247-252.

3. Paisley JW, Lauer BA, Mcintosh K, Glode MP, Schachter J, Rumack C. Pathogens associated with acute lower respiratory tract infection in young infants. Pediatric Infect Dis J 1984;3(1): 14-19.

4. Musher D. Infections caused by Streptococcus pneumoniae: clinical spectrum, pathogenesis, immunity, and treatment. Clin Infect Dis 1992; 14(4):801-809.

5. Gray BM, Converse GM III, Dillon HC Jr. Epidemiologic studies of Streptococcus pneumoniae in infants: acquisition, carriage, and infection during the first 24 months of life. J Infect Dis 1980;142(6):923-933.

6. World Health Organization, Programme for the Control of Acute Respiratory Infections. Technical bases for the WHO recomendations on the management of pneumonia in children at first level health facilities. Geneva: WHO; 1991 (WHO/ARI/91.20).

7. World Health Organization. Antibiotics in the treatment of acute respiratory infections in young children. Geneva: WHO; 1991 (WHO/ ARI/90.10).

8. Klugman KP. Pneumococcal resistance to antibiotics. Clin Microbiol Rev 1990;3(2): 171-196.

9. Appelbaum PC. Antimicrobial resistance in Streptococcus pneumoniae: an overview. Clin Infect Dis 1992;15(1):77-83.

10. Mastro TD, Ghafoor A, Nomani NK, Ishaq Z, Anwar F, Granoff DM, et al. Antimicrobial resistance of pneumococci in children with acute lower respiratory tract infection in Pakistan. Lancet 1991;337(8734):156-159.

11. Mastro TD, Nomani NK, Ishaq Z, Gahfoor A, Shaakat NF, Esko E, et al. Use of nasopharyngeal isolates of Streptococcus pneumoniae and Haemopilus influenzae from children in Pakistan for surveillance for antimicrobial resistance. Pediatr Infect Dis J 1993;12(10):824-830.

12. Converse GM, Dillon HC Jr. Epidemiological studies of Streptococcus pneumoniae in infants: methods of isolating pneumococci. J Clin Microbiol 1977;5(3):293-296

13. Facklam RR, Washington II JA. Streptococcus and related catalase-negative gram-positive cocci. En: Balows A, Hausler Jr WJ, Herrmann KL, Isenberg HD, Shadomy HJ, eds. Manual of Clinical Microbiology. 5a ed. Washington DC: American Society for Microbiology; 1991: 239-248

14. National Committee for Clinical Laboratory Standards. Performance standards for antimi- crobial disk susceptibility tests. Villanova, PA: National Committee for Clinical Laboratory Standards; 1993 (NCCLS publication M2-A5).

15. Jorgensen JH, Swenson JM, Tenover FC, Ferraro MJ, Hindler JA, Murray PR. Development of interpretative criteria and quality control limits for both microdilution and disk diffusion antimicrobial susceptibility testing of Streptococcus pneumoniae. J Clin Microbiol 1994;32(10):2448-2459.

16. National Committee for Clinical Laboratory Standards. Methods for dilution antimicrobial susceptibility tests for bacteria that grow aerobically. Villanova, PA: National Committee for Clinical Laboratory Standards, 1993 (NCCLS publication M7-A2)

17. Sorensen UBS. Typing of pneumococci by using 12 pooled antisera. J Clin Microbiol 1993; 31(8):2097-2100.

18. Dean A. Epi Info V.G.6. Atlanta, GA: Centers for Disease Control and Prevention (CDC); 1994.

19. Smith T, Lehmann D, Montgomery J, Gratten M, Riley ID, Alpers MP. Acquisition and invasiveness of different serotypes of Streptococcus pneumoniae in young children. Epidemiol Infect 1993;111(1):27-39.

20. Mogdasy M, Camou T, Fajardo C, Hortal M. Colonizing and invasive strains of Streptococcus pneumoniae in Uruguayan children: type distribution and patterns of antibiotic resistance. Pediatr Infect Dis J 1992;11:648-652.

21. Hendley JO, Sande MA, Stewart P. Spread of Streptococcus pneumoniae in families. I, Carriage rates and distribution of types. J Infect Dis 1975;132(1):55-61.

22. Lankinen KS, Leinonen M, Tupasi TE, Haikala R, Ruutu P. Pneumococci in nasopharyngeal samples from Filipino children with acute respiratory infections. I Clin Microbiol 1994;32(12):2948-2952.

23. Montgomery JM, Lehmann $D$, Smith $T$ Michael A, Joseph B, Lupiwa T, et al. Bacterial colonization of the upper tract and its association with acute lower tract infections in highland children of Papua, New Guinea. Rev Infect Dis 1990;12(Suppl 8):S1006-1016.

24. Muñoz N, Sanin J, Monroy F. Portadores nasofaríngeos de Haemophilus influenzae, Streptococcus pneumoniae, Neisseria meningitidis y Moraxella catarrhalis en una población menor de 3 años. Actualizaciones Pediátricas Fundación Santafé de Bogotá 1994;4(4):159-164.

25. Pérez J, Liñares J, Bosh J. Antibiotic resistance of Streptococcus pneumoniae in childhood carriers. I Antimicrob Chemother 1987;19(2): 278-280.

26. Robins-Brownw RM, Kharsany BM, Koornhof HJ. Antibiotic-resistant pneumococci in hospitalized children. J Hyg 1984;93(1):9-16.
27. Muñoz R, Coffey TJ, Daniels M, Dowson CG, Laible G, Casal J, et al. Intercontinental spread of a multiresistant clone of serotype 23F Streptococcus pneumoniae. I Infect Dis 1991;164(2): 302-306.

28. Klugman KP, Coffey TJ, Smith A, Wasas A, Meyers M, Spratt BG. Cluster of an erythromycin-resistant variant of the Spanish multiply resistant $23 \mathrm{~F}$ clone of Streptococcus pneumoniae in South Africa. Eur J Clin Microbiol Infect Dis 1994;13(2):171-174.

29. Calderón-Jaimes E, Echániz-Avilés G, CondeGonzález C, Rivera-Sánchez R, BarrigaAngulo G, Solórzano-Santos F, et al. Resistencia y serotipificación de 83 cepas de Streptococcus pneumoniae aisladas en niños portadores asintomáticos y enfermos. Bol Med Hosp Infant Mex 1993;50(12):854-860.

30. Koornhoof HJ, Wasas A, Klugman K. Antimicrobial resistance in Streptococcus pneumoniae: a South African perspective. Clin Infect Dis 1992;15(1):84-94

31. Sessegolo JF, Levin AS, Levy CE, Asensi M, Facklam RR, Teixeira LM. Distribution of serotypes and antimicrobial resistance of Streptococcus pneumoniae strains isolated in Brazil from 1988 to 1992. J Clin Microbiol 1994; 32(4):906-911.

32. Jorgensen J, Doern G, Maher L, Howell A Redding J. Antimicrobial resistance among respiratory isolates of Haemophilus influenzae, Moraxella catarrhalis and Streptococcus pneumoniae in the United States. Antimicrob Agents Chemother 1990;34(11):2075-2080.

33. Hansman D, Morris S. Pneumococcal carriage among children in Adelaide, South Australia. Epidemiol Infect 1988;101(2):411-417.

34. Geslin P, Buu-Hoi A, Fremaux A, Acar JF. Antimicrobial resistance in Streptococcus pneumoniae: An epidemiological survey in France, 1970-1990. Clin Infect Dis 1992;15(11):95-98.

35. Fenoll A, Bourgon CM, Muñoz R, Vicioso D, Casal J. Serotype distribution and antimicrobial resistance of Streptococcus pneumoniae isolates causing systemic infections in Spain 1979-1989. Rev Infect Dis 1991;13(1):56-60.

36. Nava JM, Bella F, Garau J, Lite J, Morera MA, Marti C, et al. Predictive factors for invasive disease due to penicillin resistant Streptococcus pneumoniae: a population-based study. Clin Infect Dis 1994;19(5):884-890.

Manuscrito recibido el 14 de febrero de 1996 y aceptado para publicación en versión revisada el 10 de julio de 1996. 
ABSTRACT Streptococcus pneumoniae is one of the principal causal agents of acute respiratory infection (ARI) in children, and its resistance to antibiotics has increased worldwide. This study examined the patterns of susceptibility to antibiotics of S. pneumoniae that

Antibiotic susceptibility of Streptococcus pneumoniae colonizing the nasopharynx of Colombian children with pneumonia had colonized the upper respiratory tract of 272 children hospitalized for pneumonia in two hospitals in Santafé de Bogotá. S. pneumoniae was isolated from 114 patients $(42 \%)$. Diminished susceptibility to penicillin was noted in 19 isolations $(17 \%)$, with $12(11 \%)$ showing an intermediate level of sensitivity and $7(6 \%)$ outright resistance. Only 1 of the 19 isolates resistant to penicillin also showed resistance to ceftriaxone. There was diminished sensitivity to erythromycin in 3 isolations (3\%), to chloramphenicol in $6(5 \%)$, and to cotrimoxazole (trimethoprim + sulfamethoxazole) in 46 $(40 \%)$. Resistance to multiple drugs was found in 7 isolations $(6 \%)$. The most commonly encountered penicillin-resistant serotype was $23 \mathrm{~F}(68.4 \%)$. An association was observed among age, previous use of antibiotics, and colonization by S. pneumoniae with reduced penicillin susceptibility or multiple-drug resistance. This study confirmed the presence of antibiotic-resistant S. pneumoniae in Colombia and highlights the importance of the rational use of antibiotics and of the implementation of epidemiologic surveillance for this agent.

\title{
Congreso Centroamericano de Microbiología XI Congreso Internacional
}

\author{
Fechas: 23 a 27 de noviembre de 1997 \\ Lugar: $\quad$ Ciudad de Guatemala, Guatemala \\ Tema: $\quad$ Microbiología clínica y aplicada
}

El Instituto de Investigaciones del Centro de Estudios en Salud, Universidad del Valle de Guatemala, y el Instituto de Nutrición de Centro América y Panamá (INCAP), Organización Panamericana de la Salud, organizan este congreso, en el cual se tratarán temas importantes relacionados con los campos de la microbiología, la biotecnología, el medio ambiente y la salud, entre otros.

\author{
Información: \\ Licda. Olga Torres \\ INCAP \\ Apartado postal 1188 \\ 01901 Guatemala, Guatemala \\ Teléfonos: (502) 4723762 a 67; Fax: (502) 4736529 \\ Correo electrónico: otorres@incap.org.gt \\ WWW, http://www.uvg.edu.gt/noticias/ccamicro
}

